\title{
Der Arzt, das Baby und das Kuscheltuch
}

\author{
Rolf Adler
}

Kehrsatz

\section{Einleitung}

Unter Übergangsobjekt verstehen wir ein Kuscheltuch, ein Stofftier, das teilnimmt an der frühesten Beziehung zwischen Mutter und Säugling/Kleinkind, und die Mutter während ihrer Abwesenheit ersetzen kann.

Anhand einiger Arzt-Patient/in-Interaktionen möchte ich beleuchten, wie sich Blockierungen in der Therapie zeigen und sich durch den Einsatz von Übergangsobjekten vielleicht beeinflussen lassen.

1. Eine 60 -jährige Frau liess sich gegen ihre von ihrem Ehemann verlangte Einwilligung in die Scheidung von ihrem notorisch fremdgehenden Mann scheiden. Dann erlitt sie eine Knieverletzung beim Skifahren. Sie wurde nie mehr schmerzfrei. Die Knieschmerzen zogen in den nächsten Jahren acht Operationen nach sich. Die Schmerzen breiteten sich auf den ganzen Körper aus. Schwindel, grosse Müdigkeit, Druck auf der Brust, Rücken- und Bauchschmerzen prägen das derzeitige Bild. Ein Orthopäde, der keine Operationsindikation sah, wies mir die Patientin zu. Ich sehe sie seit anderthalb Jahren wöchentlich für 50 Minuten.

Die Patientin arbeitet mit grösster Willensanstrengung noch halbtags, liegt in der restlichen Zeit zuhause. Ihre Jugend war gekennzeichnet vom Alkoholismus des Vaters mit Vernachlässigung der Familie, von schwerem Missbrauch, auch intim durch ältere Brüder, von fehlender Unterstützung für eine höhere Schulbildung trotz guter Intelligenz und guten Schulleistungen.

Sie kommt gern und pünktlich von auswärts in die Sprechstunde, zeigt eine Art Verständnis für eine Beziehung zwischen ihrem Zustand und ihrer «pain-prone»-Jugend [1], und doch bewirken diese verstandesmässigen Einsichten keine Veränderung ihres Leidens. An der frühen Kindheit lasse sich jetzt nichts mehr ändern, sie sei selbst schuld, dass sie als Kind so geplagt worden sei. In einer Sitzung nach anderthalb Jahren fühlt sie sich erbärmlich, total erschöpft, am ganzen Körper eingehüllt in brennende Schmerzen. Enorm schlimm sei, dass ihr niemand glaube, wie sehr sie leide.

Wir sitzen uns an der Ecke eines kleinen Tischchens gegenüber. Ich: «Ich bin ein grosser Elefant mit grossen Ohren, so gross, dass dieses Ohr Sie ganz einwickeln kann.» Sie: «Das geht doch gar nicht.» Ich nehme ein Papiertaschentuch, einen daumenballengrossen kristall- haltigen Stein vom Tischchen, den mir eine Walliser Patientin aus dem Binntal geschenkt hat, wickle ihn ein und drücke ihn der Patientin in die Hand. Sie nimmt ihn entgegen und äussert erleichtert: «Genau so habe ich es mit meiner Tochter gemacht, meinem einzigen Kind!» Sie lächelt, es ist Ende der Stunde, wir verabschieden uns.

2. Eine Kollegin bittet mich, die 75-jährige, ehemalige Klassenkameradin als Patientin anzunehmen. Sie sei schon zweimal mehrere Wochen in Kliniken gewesen. Ihre Schwindelzustände, Panikattacken, Herzrasen und Schlafstörungen hielten an, dazu habe sie Mühe beim Atmen mit Verengungsgefühl im Kehlkopfbereich. Zudem sei sie einsam, halte das Alleinsein nicht aus.

Ich sehe die Patientin zweimal pro Woche. Als sie vier Jahr alt war, sei ihre Mutter an Polio erkrankt und an Erstickung rasch gestorben. Sie habe sich zuhause immer einsam gefühlt. Im Gymnasialalter habe sie erwogen, auszuziehen und allein zu leben. Sie habe Ökonomie studiert und später bei mehreren Ausland-Hilfsprojekten mitgearbeitet, Schulen in Südamerika gegründet, geholfen, dort Klöster aufzubauen und Flüchtlinge betreut. Sie reagiert auf meine sachten Bemerkungen, dass ihre heutige Panik und Einsamkeit eine Geschichte haben müssen. Auf eine mögliche Verbindung zwischen ihrer Atemnot und dem Erstickungstod der Mutter kann sie einen Blick werfen, zieht ihn aber rasch zurück, betont ihre jetzige schlimme Situation und bittet um rasche Hilfe. In den ersten Wochen unserer Gespräche sind nächtliche telefonische Hilferufe keine Ausnahme.

Sie drängt darauf, eine Knieoperation wegen Arthrose vornehmen zu lasssen. Ich bin skeptisch und möchte zuerst eine Beruhigung in der Gesprächstherapie beobachten, aus der Befürchtung heraus, die Schmerzen könnten trotz Eingriff anhalten. Zwei Sitzungen vor der Operation berichtet sie mir zu Beginn der Stunde, sie sei nach dem letzten Gespräch beim Verlassen der Praxis von Weinen überrascht worden. Ob sie Worte dafür finden könne, frage ich sie. Ja, ich hätte unter der Tür beim Abschied meine Hand auf ihre Schulter gelegt. Ihre Einsamkeit sei ihr in jenem Moment besonders nahegekommen.

Am Tag vor der Operation besuche ich sie im Krankenhaus, lege ein kleines Buch [2] auf ihren Nachttisch und sage dazu, der vierte Abschnitt im Buch beinhalte eine Sage aus dem 12. Jahrhundert in Norwegen (wo der fein- 
fühlige König nach mehreren Versuchen, seinen isländischen Hofgast mit Ratschlägen und Angeboten aus der Depression heraus führen, scheitert, Gespräche über mehrere Wochen am Kaminfeuer im Königspalast aber zur Aufhellung des Hofdichters führen). Ich weise lediglich darauf hin, wo diese Sage im Buch zu finden sei, gehe aber nicht auf den Inhalt ein. Nach der Operation besuche ich die Patientin zweimal. Aus dem Rehabilitationsaufenthalt berichtet sie mir am Telefon, das Büchlein sei für sie zu komplex. Einen Tag später ruft sie an, ihr Zimmer sei jetzt viel schöner und sonnig - welchen Abschnitt im Büchlein sie lesen solle. Sie klingt aufgeräumt und telefoniert nicht so lange wie noch vor Wochen. Einen Tag später hat sie die Sage gelesen und äussert, dass sie verstehe, was ich ihr sagen will.

3. Einer meiner früheren Oberärzte bittet mich zur Supervision. Diesmal lädt er einen 40-jährigen Mann ein und bittet mich, diesen in seiner Anwesenheit zu interviewen. Ein flotter Mann in guter Haltung sitzt mir gegenüber. Er sei verspannt, unsicher in der Körpermotorik und leide unter Zwangsgedanken. Er bewegt wiederholt den Kopf in verschiedene Richtungen, um seine Halsmuskeln zu lockern. Die Gedanken kreisen um seine Arbeit in einer Informatikfirma. Er als Leiter seiner Abteilung käme mit seinen Mitarbeitern gut voran, die Firma gehe aber wegen der versagenden übrigen Abteilungen bald bankrott. Er gebe sich ausserordentlich Mühe. Bisher seien ihm im ganzen Leben nie Schwierigkeiten oder Fehler unterlaufen. Seit der Kindheit sei er der Fels seiner Eltern und einer schwierigen Schwester. In der jetzigen Lage sei er aber verzweifelt und hilflos. Er sorge sich um seine Familie, weil er gekündigt habe und an der neuen Arbeitsstelle nur die Hälfte seines jetzigen Verdienstes erhalten werde. Ich frage ihn, ob noch andere Gefühle seine Hilflosigkeit begleiten würden. Er wägt ab, hin und her, äussert aber keine zusätzlichen. Ich: «Ich vermute, Sie schämen sich.» Er bejaht seufzend.

Ich übernehme den Patienten und sehe ihn zweimal wöchentlich. Er ist erstaunt, dass ich bei der zweiten Sitzung den Vornamen seiner Frau, seiner drei Kinder, seiner zwei Hunde und seines Katers erinnere. Ich erkläre ihm, dass ich mich in seine Situation gleiten lasse, dann sei das Erinnern selbstverständlich. In einer der nächsten Sitzungen ist er weniger verspannt, aber immer noch deutlich motorisch ungeschickt. Ich drücke ihm meinen Vierfarbenstift in die Hand, den er beim Aufstehen am Ende der Stunde fallen lässt, mit zittriger Hand aufhebt und in seine Tasche gleiten lässt. Ich: «Er ist jetzt bei Ihnen, begleitet Sie und am Ende unserer Therapie geben Sie, ihn mir zurück.»

4. Meine fünf Jahre Psychoanalyse mit vier Stunden pro Woche verliefen eher enttäuschend. Die Analytikerin muss theoretisch sehr bewandert gewesen sein, eine gewisse Wärme aber fehlte mir bei ihr. An einem Wintertag war es auf der Couch kalt. Mitten in der Stunde ging die Analytikerin zu einem Schrank, holte eine Decke hervor, kam zur Couch und breitete die Decke über mich aus. Diese Szene bleibt mir am deutlichsten haften bis heute, nach über 40 Jahren.

\section{Überlegungen}

Bei allen vier Handlungen - Hand auf Schulter, eingewickelter Kristall, Vierfarbenstift und Decke - agierte ich, oder wurde mit mir agiert, d.h. eine Handlung durchgeführt. Dabei wurden keine Worte gewechselt. Woher ahnte ich, bzw. die Analytikerin, was zu tun war? Es ist die Einfühlung (Empathie) ins Gegenüber, in psychoanalytischer Sprache ein Element der Gegenübertragung. Ob die vier Handlungen günstig waren, lässt sich erst am Verlauf der Therapien beurteilen. Wichtig ist, die Patientinnen und Patienten zu fragen, wie sich diese Momente anfühlten. Die Betonung in der Psychoanalyse, auf keinen Fall einzugreifen, darf m.E. in jeder Episode neu überlegt werden.

Hier sei an den berühmten Mathematiker Charles S. Peirce [2, 3] erinnert, der ein Erlebnis schildert, bei dem die Empathie in der Gegenübertragung eine Rolle spielte: Er war per Schiff von Boston nach New York gereist. Ausgestiegen stellte er noch auf dem Pier fest, dass er seine goldene Uhr, ein Geschenk des US-Präsidenten für seine dem Land erwiesenen Dienste, in der Kabine vergessen hatte, aber es war zu spät, um dorthin zurückzugehen. Dank seiner Berühmtheit konnte er den Kapitän veranlassen, alle Matrosen auf Deck zu beordern und sich in einer Reihe aufzustellen. Peirce schritt die Reihe von Mann zu Mann ab, jeden in ein kurzes, banales Gespräch verwickelnd. Er kam sich dabei läppisch vor. Dann ging er zur Seite, tauchte in seine Gefühlswelt ein und stellte fest, dass ihm ein Matrose besonders aufgefallen war. Er liess diesen Mann durch einen Privatdetektiv beschatten in der Annahme, dieser unbemittelte Mann werde die Uhr zum nächsten Pfandleihgeschäft im Hafen tragen, um sie in Geld umzusetzen. Peirce hatte richtig vermutet. Die Uhr fand sich dort. Spannend ist Peirces Anmerkung, dass ihm dieses Vorgehen schon oft beim Lösen mathematischer Probleme weitergeholfen habe. In der Sprache der Kindheitsentwicklung hat er sich einen Spielraum geschaffen, in dem Fantasie und schöpferische Tätigkeit möglich werden.

Handlungen, die sich in unseren Gesprächen mit Patientinnen und Patienten einstellen, averbal stattfinden und intuitiv genannt werden können, hängen vermutlich mit frühkindlichen Zuständen zusammen, in denen nicht unsere Signal-Prüfaffekte [4] (Anflug von Ärger, Anflug von Schuld usw.) die Führung übernehmen, sondern die Trieb-Entladungsaffekte (heftige Wut, hilflose Leere usw.), bei deren Funktionen die nicht-dominante Hemisphäre die Hauptrolle übernimmt.

Damit befinden wir uns im Säuglings- und Kleinkindesalter: Hier gibt es nicht einerseits eine Mutter und andererseits einen Säugling, sondern den Funktionskreis, in dem beide eingeschlossen sind. Spürt der Säugling ein Bedürfnis, etwa nach Nahrung, wird er unruhig, vielleicht weint er (er wirkt). Die genügend gute Mutter [5] bemerkt die Veränderung beim Baby, gibt ihr eine Bedeutung. In der Zeichenlehre (Semiotik, s. [3]) würde man sagen: Die Mutter prägt einer Veränderung in ihrer Umgebung ein Merkmal auf, das Baby-Verhalten und das Merkmal werden zum Zeichen [6]. Dieses wird im Ich der Mutter, hier 
Interpretantin genannt, verarbeitet. Nach der Verarbeitung handelt die Mutter, sie wirkt. Die zweigliedrige Ursache-Wirkungskette wird dadurch dreigliedrig - eine Ursache wird bemerkt - die Interpretantin plant - und veranlasst ein Wirken, eine Handlung. Im Lauf der Entwicklung macht das Baby viele Erfahrungen, beispielsweise beim Gestilltwerden, wenn es in den Arm genommen wird, den Duft der Mutter registriert, den Geschmack der Milch, averbale Laute der Mutter usw. Es verinnerlicht diese Erfahrungen. Es entsteht die verinnerlichte Mutter. Zur Interaktion kann etwa ein Kuscheltuch oder ein Stofftier kommen. Das Tuch wird in den Mund genommen, gesaugt, sein Duft nach der Mutter wahrgenommen. Befriedigt die Mutter ein Bedürfnis des Säuglings nicht sofort, greift das Baby nach dem Tuch, das die Abwesenheit der Mutter ersetzt. Das Tuch (Objekt) ist Übergangsobjekt geworden. Es repräsentiert die Mutter und wird unabhängig von ihr zu einer verinnerlichten Erinnerung an sie. Damit wird der Säugling schrittweise unabhängiger.

Ich vermute, dass die Hand auf der Schulter, der Stein, der Vierfarbenstift und die Decke Eigenschaften des Übergangsobjekts aufweisen. In kritischen Lebensphasen des Erwachsenen neigt dieser zur Regression (Rückkehr in frühe Lebensphasen). Worte, Empfehlungen, Ratschläge und Verhaltensanweisungen bringen in solchen Situationen wenig bis nichts. Das Übergangsobjekt lebt auf mit den mit ihm verbundenen Emotionen. Sie können mit der Patientin/dem Patienten sachte in das Gespräch aufgenommen werden, sodass die Patientin/der Patient erahnen und schliesslich verstehen kann, weshalb gewisse Konflikte zu Körpersymptomen geführt haben. In bestimmten Momenten vermag das Übergangsobjekt zu beruhigen, die Hilf- und Hoffnungslosigkeit mildern, sodass die reiferen Teile des Ichs die Führung wieder übernehmen können.

Amulette, Kreuzlein, Spangen, Kettchen besitzen auch Eigenschaften eines Übergangsobjekts, ebenso wie die Ärztin/der Arzt selbst. Und damit wären wir bei Michael Balints «Droge Arzt» [7] angelangt. Die Droge Ärztin/Arzt ist ein komplexes Gebilde: Sie/er ist reale(r) Ansprechpartner/in, mit der/dem reale Abmachungen wie Abklärungen, Labortests, Eingriffe usw. abgesprochen werden.
Dann wird sie/er zusätzlich mit Erwartungen, Wünschen, Befürchtungen, Wesenszügen früherer Bezugspersonen der Patientin/des Patienten ausgestattet, dazu gehören eben auch Erlebnisse, die im Unbewussten der Patientin/ des Patienten gespeichert, auf die Ärztin/den Arzt übertragen werden.

\section{Nachtrag}

In «Der Bund» vom Samstag, dem 11. April 2020 schreibt eine Tänzerin, die sich in unserer Corona-Zeit einer Wahloperation unterziehen musste: «Zum Glück war - wie in vielen Krankenhäusern derzeit - extrem wenig los, sodass sich die Leute viel Zeit für mich nehmen konnten. Es waren auch alle sehr nett zu mir. Als ich von der Narkose aufgewacht bin, haben sich zahlreiche Pflegende gleich um mich gekümmert. Speziell aufgefallen ist mir eine Frau, die mir über den Arm gestreichelt hat. Ich habe in diesem Moment wieder realisiert, wie wichtig Körperkontakt ist und wie sehr er derzeit Mangelware ist.»

\section{Bibliografie}

1. Engel GL. Psychogenic pain and the pain-prone patient. Am J Med. 1959:26:899-918.

2. Adler RH. Herausforderung für die Biomedizin: Das Biopsychosoziale Konzept. Muttenz; EMH: 2017.

3. Peirce CS. What is a sign. In: Buchler J et al.: Philosophic writings of Peirce. New York; Dower: 1955. 98-101.

4. Engel GL. Psychologial development in health and disease. Philadelphia; Saunders: 1962.

5. Winnicott D. Reifungsprozesse und fördernde Umwelt. München; Kindler: 1975.

6. Uexküll Th v, Wesiack W. In: Adler RH et al.: Uexküll, Psychosomatische Medizin. 7. Aufl. München; Urban \& Fischer: 2010.

7. Balint M. Der Arzt, sein Patient und die Krankheit. Hamburg; Fischer: 1970.

Prof. em. Dr. med. Rolf Adler

Leiserenweg 4

3122 Kehrsatz

michele.rolf.adler@gmail.com 